\title{
A Nitrogen-Walk Approach to Explore Bioisosteric Replacements in a Series of Potent A2B Adenosine Receptor Antagonists
}

Ana Mallo-Abreu. ${ }^{1,2}$ Rubén Prieto-Díaz, ${ }^{1,2}$ Willem Jespers, ${ }^{3}$ Jhonny Azuaje, ${ }^{1,2}$ Maria Majellaro, ${ }^{1,2}$ Carmen Velando, ${ }^{1}$ Xerardo García-Mera,${ }^{2}$ Olga Caamaño, ${ }^{2}$ José Brea, ${ }^{4}$ María I. Loza, ${ }^{4}$ Hugo Gutiérrez-de-Terán ${ }^{3 *}$ and Eddy Sotelo ${ }^{1,2 *}$

${ }^{1}$ Centro Singular de Investigación en Química Biolóxica y Materiais Moleculares (CIQUS),

${ }^{2}$ Departamento de Química Orgánica, Facultade de Farmacia, Universidade de Santiago de Compostela, 15782 Santiago de Compostela, Spain. ${ }^{3}$ Department of Cell and Molecular Biology, Uppsala University, Uppsala SE-75124. ${ }^{4}$ Centro Singular de Investigación en Medicina Molecular y Enfermedades Crónicas (CIMUS), Universidade de Santiago de Compostela, 15782 Santiago de Compostela, Spain.

\section{TABLE OF CONTENTS}

Calculated free energies tables $\mathrm{S} 2-\mathrm{S} 3$

HPLC traces of ligands 18g (ISAM-C032), 17e (ISAM-A344) and 17f (ISAM-A275). . . . S4 
Table 1: Calculated free energies $(\mathrm{kcal} / \mathrm{mol})$ between two rotamers of the R4 position of ISAM140, ISAM-141 and ISAM-C032.

\begin{tabular}{lcr||r} 
Compound & \multicolumn{1}{c||}{$\boldsymbol{\Delta} \mathbf{G}_{\text {protein }}$} & \multicolumn{1}{c|}{$\Delta \mathbf{G}_{\text {water }}$} & \multicolumn{1}{c}{$\boldsymbol{\Delta} \mathbf{\Delta}$} \\
\hline \hline ISAM-140 & $-0.34 \pm 0.13$ & $-0.42 \pm 0.06$ & $0.08 \pm 0.15$ \\
\hline ISAM-141 & $0.10 \pm 0.19$ & $-0.01 \pm 0.19$ & $0.11 \pm 0.18$ \\
\hline ISAM-C032 & $0.25 \pm 0.15$ & $0.22 \pm 0.14$ & $0.03 \pm 0.15$ \\
\hline
\end{tabular}


Table 2: Calculated and experimental relative binding free energies $(\mathrm{kcal} / \mathrm{mol})$ of al compounds within the series. to ISAM-140.

\begin{tabular}{|c|c|c|c|c|c|c|c|}
\hline Compound & $\Delta G_{\text {protein }}$ & SEM & $\Delta \mathrm{G}_{\mathrm{water}}$ & SEM & $\Delta \Delta \mathrm{G}_{\mathrm{calc}}$ & SEM & $\Delta \Delta \mathrm{G}_{\exp }$ \\
\hline ISAM140 & -- & -- & -- & -- & -- & -- & -- \\
\hline $17 a$ & 24.63 & 0.44 & 28.34 & 0.61 & -3.71 & 0.74 & -3.21 \\
\hline $17 \mathrm{~b}$ & 24.32 & 0.54 & 26.20 & 0.14 & -1.88 & 0.48 & -3.16 \\
\hline $17 \mathrm{c}$ & 8.92 & 0.63 & 12.43 & 0.55 & -3.51 & 0.83 & -3.14 \\
\hline $17 d$ & 8.81 & 0.20 & 10.68 & 0.16 & -1.87 & 0.25 & -3.41 \\
\hline $17 \mathrm{e}$ & -20.01 & 0.31 & -17.05 & 0.45 & -2.96 & 0.54 & -1.13 \\
\hline $17 f$ & -20.56 & 0.30 & -18.77 & 0.31 & -1.79 & 0.43 & -1.27 \\
\hline $17 \mathrm{~g}$ & -8.16 & 0.47 & -6.89 & 0.65 & -1.27 & 0.79 & $* \leq-3.60$ \\
\hline $17 \mathrm{~h}$ & -10.44 & 0.15 & -8.67 & 0.11 & -1.77 & 0.18 & -2.59 \\
\hline $17 i$ & -14.85 & 0.52 & -10.94 & 0.48 & -3.91 & 0.71 & -1.94 \\
\hline $17 \mathrm{j}$ & -13.62 & 0.08 & -12.77 & 0.18 & -0.85 & 0.18 & -2.71 \\
\hline $17 \mathrm{k}$ & 19.01 & 0.35 & 21.74 & 0.65 & -2.73 & 0.71 & -3.15 \\
\hline $17 \mid$ & 18.19 & 0.45 & 19.79 & 0.14 & -1.60 & 0.42 & -3.37 \\
\hline $17 m$ & 18.08 & 0.51 & 19.97 & 0.18 & -1.89 & 0.49 & -1.98 \\
\hline $17 n$ & -0.27 & 0.16 & 0.25 & 0.13 & -0.52 & 0.21 & -2.32 \\
\hline $18 \mathrm{a}$ & 24.81 & 0.39 & 26.45 & 0.52 & $\begin{array}{l}-1.64 \\
\end{array}$ & 0.64 & -3.19 \\
\hline $18 \mathrm{~b}$ & 23.71 & 0.26 & 24.80 & 0.16 & -1.09 & 0.30 & -3.15 \\
\hline $18 \mathrm{c}$ & 14.03 & 0.58 & 13.58 & 0.56 & 0.45 & 0.81 & -2.79 \\
\hline $18 \mathrm{~d}$ & 12.10 & 0.15 & 11.72 & 0.13 & 0.38 & 0.20 & -3.18 \\
\hline $18 \mathrm{e}$ & 20.55 & 0.63 & 23.94 & 0.53 & -3.39 & 0.82 & -2.81 \\
\hline $18 \mathrm{~g}$ & 11.34 & 0.46 & 12.52 & 0.55 & -1.18 & 0.71 & -0.50 \\
\hline $18 \mathrm{~h}$ & 9.84 & 0.15 & 11.39 & 0.10 & -1.55 & 0.18 & -1.49 \\
\hline $18 \mathrm{i}$ & 16.78 & 0.22 & 17.16 & 0.46 & -0.38 & 0.48 & $\leq-3.60$ \\
\hline $18 \mathrm{j}$ & 15.12 & 0.43 & 15.23 & 0.14 & -0.11 & 0.40 & $\leq-3.60$ \\
\hline $18 \mathrm{k}$ & -3.05 & 0.76 & 0.59 & 0.44 & -3.64 & 0.85 & $\leq-3.60$ \\
\hline 181 & -5.78 & 0.34 & -1.92 & 0.21 & -3.86 & 0.39 & $\leq-3.35$ \\
\hline $18 \mathrm{~m}$ & -3.49 & 0.37 & -1.10 & 0.58 & -2.39 & 0.67 & -2.95 \\
\hline $18 n$ & -3.55 & 0.23 & -3.09 & 0.12 & -0.46 & 0.25 & $\leq-3.60$ \\
\hline 180 & -10.77 & 0.44 & -10.28 & 0.42 & -0.49 & 0.61 & -2.44 \\
\hline $18 p$ & -12.02 & 0.19 & -12.02 & 0.14 & 0.00 & 0.23 & -1.76 \\
\hline
\end{tabular}

* $\leq$ indicates that the substituted compound had a Ki lower than the affinity measurement threshold. 
Waters

Project Name jhonny

Reported by User: Breeze user (Breeze)

\section{SAMPLE INFORMATION}

Sample Name: ISAM-CO32

Sample Type: Unknown

Vial: $\quad 1$

Injection \#: $\quad 1$

Run Time: $\quad 35,00$ Minutes
Acquired By:

Breeze

Sample Set Name:

Acq. Method: $\quad 30 \%$ IPA Hexano

Date Acquired: $\quad$ 29/05/2020 16:44:20 CET

Injection Volume: 20,00 ul

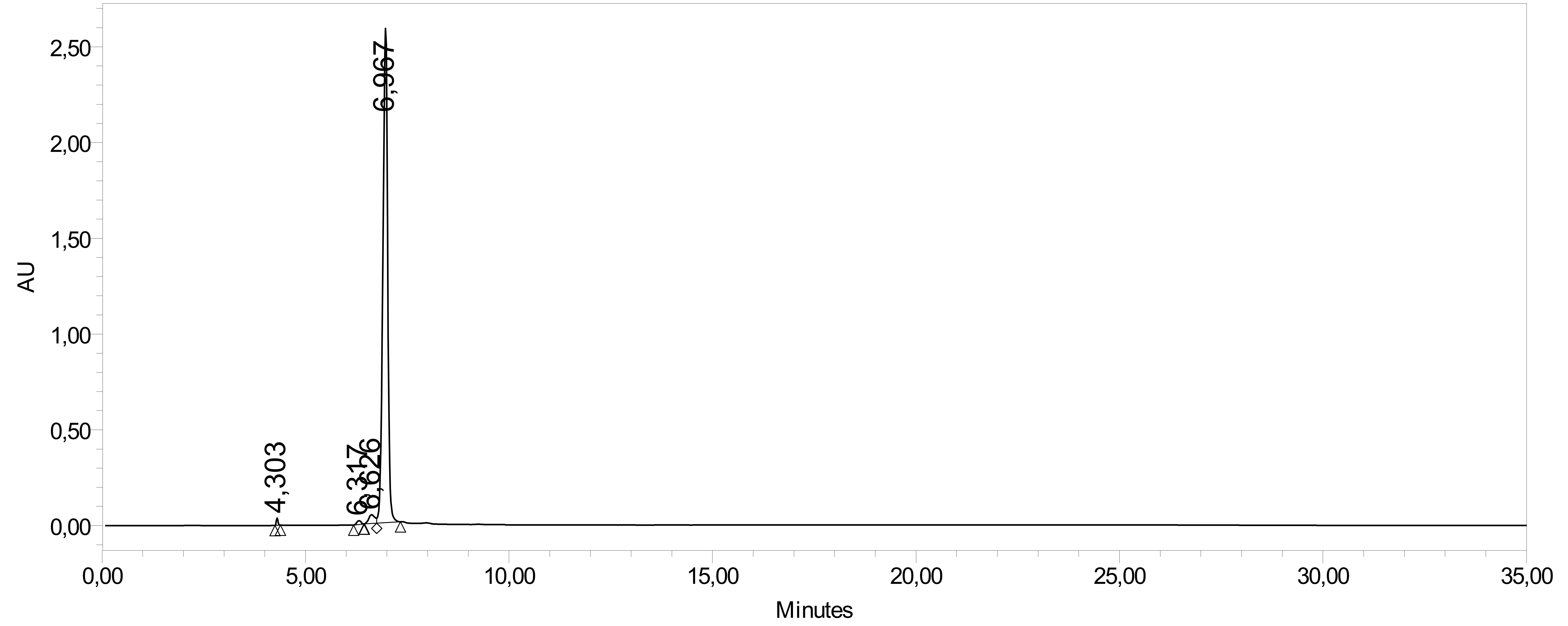

Channel: 2487Channel 1; Channel Desc.: ; Processing Method: 30\%IPA HEXANO

\begin{tabular}{|r|c|r|r|r|}
\hline & $\begin{array}{c}\mathrm{RT} \\
(\mathrm{min})\end{array}$ & $\begin{array}{c}\text { Area } \\
\left(\mu \mathrm{V}^{*} \mathrm{sec}\right)\end{array}$ & $\%$ Area & \multicolumn{1}{c|}{$\begin{array}{c}\text { Height } \\
(\mu \mathrm{V})\end{array}$} \\
\hline 1 & 4,303 & 104347 & 0,48 & 34720 \\
\hline 2 & 6,317 & 138848 & 0,63 & 19835 \\
\hline 3 & 6,626 & 481380 & 2,19 & 45403 \\
\hline 4 & 6,967 & 21233647 & 96,70 & 2576164 \\
\hline
\end{tabular}


Waters

Project Name jhonny

Reported by User: Breeze user (Breeze)

\section{SAMPLE INFORMATION}

Sample Name:

Sample Type:

Vial:

Injection \#:

Run Time:

ISAM-A344

Unknown

1

1

30,00 Minutes
Acquired By:

Breeze

Sample Set Name:

Acq. Method: $\quad 30 \%$ IPA Hexano

Date Acquired: $\quad$ 29/05/2020 19:08:58 CET

Injection Volume: 20,00 ul

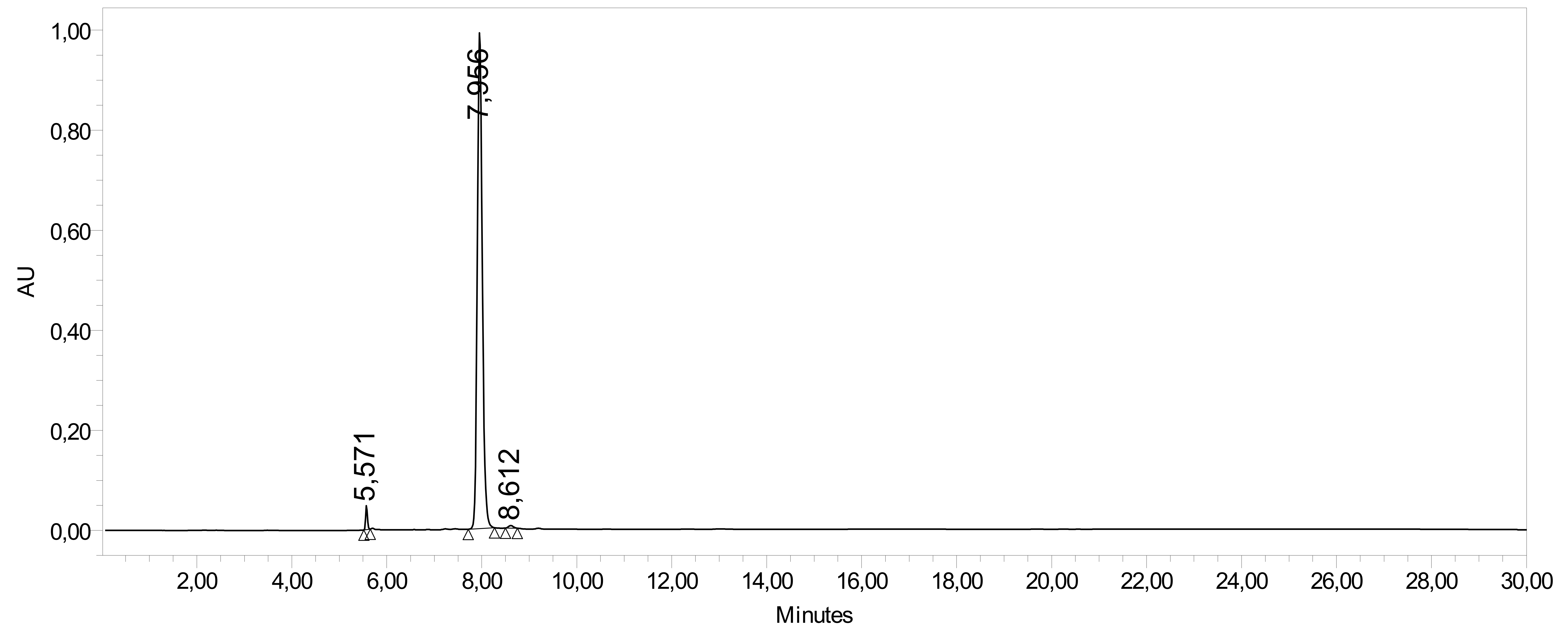

Channel: 2487Channel 1; Channel Desc.: ; Processing Method: 30\%IPA HEXANO

\begin{tabular}{|l|c|r|r|r|}
\hline & $\begin{array}{c}\text { RT } \\
(\mathrm{min})\end{array}$ & $\begin{array}{c}\text { Area } \\
\left(\mu \mathrm{V}^{*} \mathrm{sec}\right)\end{array}$ & $\%$ Area & \multicolumn{1}{c|}{$\begin{array}{c}\text { Height } \\
(\mu \mathrm{V})\end{array}$} \\
\hline 1 & 5,571 & 132366 & 1,78 & 43660 \\
\hline 2 & 7,956 & 7279488 & 97,73 & 993131 \\
\hline 3 & 8,612 & 36941 & 0,50 & 5116 \\
\hline
\end{tabular}


Waters

Project Name jhonny

Reported by User: Breeze user (Breeze)

\section{SAMPLE INFORMATION}

Sample Name:

Sample Type:

Vial:

Injection \#:

Run Time:

ISAM-A275

Unknown

1

2

30,00 Minutes
Acquired By:

Breeze

Sample Set Name:

Acq. Method: $\quad 30 \%$ IPA Hexano

Date Acquired: $\quad$ 29/02/2018 10:52:45 CET

Injection Volume: 20,00 ul

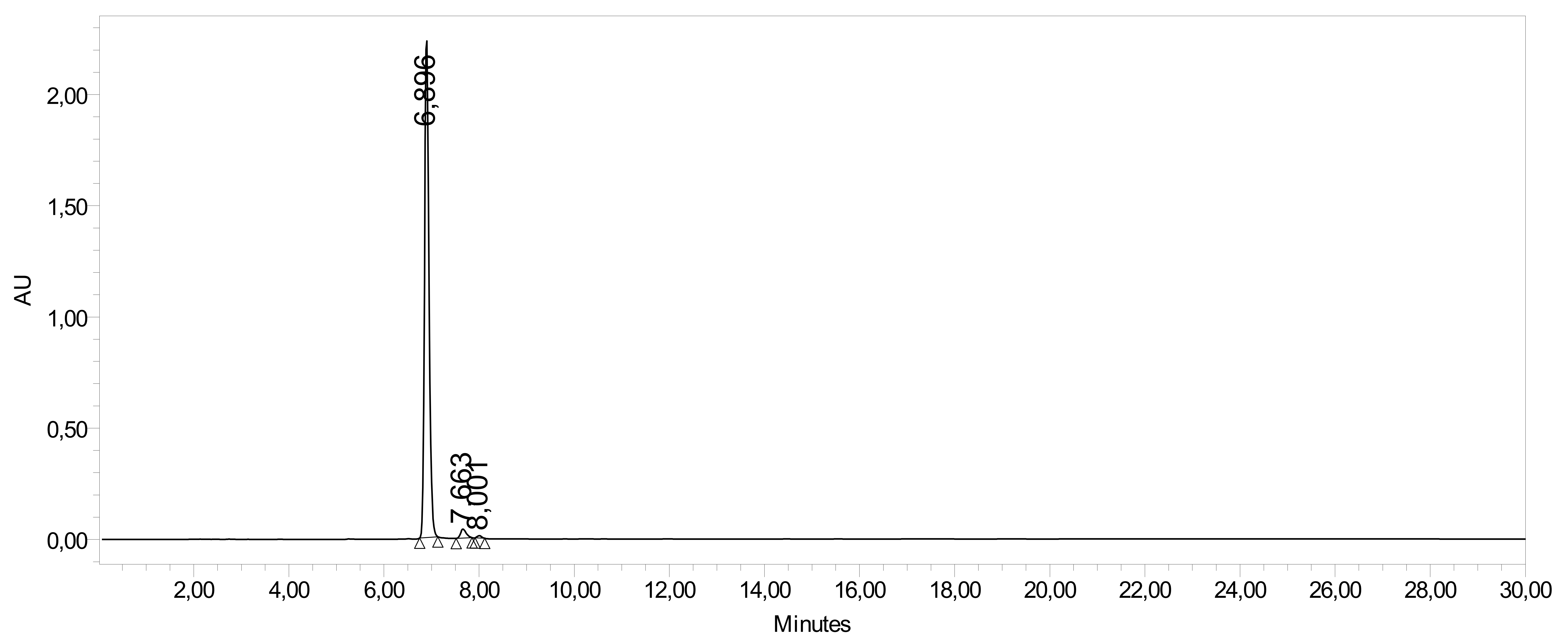

Channel: 2487Channel 1; Channel Desc.: ; Processing Method: 30\%IPA HEXANO

\begin{tabular}{|l|c|r|r|r|}
\hline & $\begin{array}{c}\text { RT } \\
(\mathrm{min})\end{array}$ & $\begin{array}{c}\text { Area } \\
\left(\mu \mathrm{V}^{*} \mathrm{sec}\right)\end{array}$ & $\%$ Area & \multicolumn{1}{c|}{$\begin{array}{c}\text { Height } \\
(\mu \mathrm{V})\end{array}$} \\
\hline 1 & 6,896 & 14506753 & 97,34 & 2235898 \\
\hline 2 & 7,663 & 330812 & 2,22 & 39551 \\
\hline 3 & 8,001 & 65219 & 0,44 & 10417 \\
\hline
\end{tabular}

